\title{
Engineer immune cells to fight cancer
}

Keywords: immune cells, cancer, tumor cells, microenvironment, fibroblast, stromal cells, endothelial cells, cancer immunotherapy, pd-1, ctla- 4 , nsclc, cscs

\section{Editorial}

Despite huge effort made in fighting the battle against cancer, it remains to be among the leading causes of death globally. According to the world health organizations, the number of new cancer cases will increase to 22 million within the next 20 years. ${ }^{1}$ Now a days, it has been widely accepted that cancer is not merely a disease of tumor cells. Various components in the tumor microenvironment, such as fibroblast, stromal cells, endothelial cells, immune cells, extracellular matrix, may affect cancer progression profoundly. Generally, immune system plays a critical role in recognizing malignant cells with distinct markers and eliminating them in a process termed immune surveillance. However, one hallmark of tumor cells is their ability to escape immune recognition, and even suppress the body's natural immune response. ${ }^{2}$ The idea of manipulating immune cells to fight cancer dates back more than a century ago. It has been shown that $\mathrm{B}$ cells as well as T cells, natural killer cells, monocytes and other immune cells could infiltrate into tumor mass and actively regulate its growth and metastasis. Extensive studies have been performed to better understand the cross talk between cancer cells and immune components. Recently, in addition to traditional treatments targeting malignant cancer cells, increasing emphasis has been put on redirecting the immune responses to eradicate tumor cells, so-called cancer immunotherapy.

During the past few decades, an increasing number of immunecell based therapies have been tested in preclinical studies and clinical trials, and brought breakthroughs with significant advances in cancer treatment. Immunotherapeutic strategies include checkpoint inhibitors, adoptive cell therapy, cancer vaccines, and artificially synthesized antibodies or antigen presenting cells. Immune checkpoint inhibitor therapy, in particular, has started a new era for cancer immunotherapy. Cancer cells trick immune cells by sending signals at certain checkpoints, such as PD-1, CTLA-4, to indicate that they are 'normal'. Checkpoint inhibitors reveal these cancer cells to be attacked by the immune system by reintroduce immune cells to recognize and terminate cancer cells, hold a great promise in the medical oncology and therapeutics. Ipilimumab was the first checkpoint inhibitor approved by the U.S. Food and Drug Administration for the treatment of metastatic melanoma in 2011. Followed by the approval of pembrolizumab and nivolumab for melanoma three years later. In the past year alone, more than ten new drugs were added to the list of immune-based anti-cancer drugs approved by the FDA and for the first time, pembrolizamab was approved for the first-line treatment of patients with metastatic non-small cell lung cancer (NSCLC). With increasing numbers of encouraging clinical trials, the application of these drugs has been highly extended to more types of cancers, including bladder cancer, ovarian cancer, lung cancer, and kidney cancers. In the available clinical studies and reports, some patients suffering from notorious highly invasive and metastatic cancers responded strikingly to immunotherapy. Reported in the New England Journal of Medicine,
Volume 2 Issue 3 - 2017

\author{
Dan Li, Su Song \\ Feinstein Institute for Medical Research, USA
}

Correspondence: Dan Li, Feinstein Institute for Medical Research, North well Health, 350 Community Dr, Manhasset, NY,USA,Email Dli7@northwell.edu

Received: March 30, 2017| Published: March 31, 2017

a 50-year-old patient with lethally recurrent brain tumor and spinal metastasis received chimeric antigen receptor (CAR)-engineered $\mathrm{T}$ cells targeting the tumor-associated antigen IL13R $\alpha 2$ intracranially for 7.5 months. After the treatment, all intracranial and spinal tumors shrunk and even disappeared temporarily. ${ }^{3}$ Although currently under treatment for recurrent brain tumor, he remains alive 15 months later after been told that he has weeks to live. Accumulating evidences point out that immunotherapy could provide improved survival, more importantly, progression-free survival. As mentioned above, immunotherapy is based on the stimulation of naturally occurring process against foreign elements. Fewer side effects were reported compared to traditional chemotherapy, which could dramatically improved the quality of patient life.

However, the current exciting advances come with challenges and setbacks. Although patients that could benefit from these treatments show remarkable responses, only a small subpopulation of patients responds to immunotherapy. ${ }^{4}$ Many questions remain to be answered. Most importantly, how to predict ahead of time which patient is going to benefit from certain immunotherapy regimens? The frustration at present is to identify biomarkers predictive of response. For a long time, researchers and doctors have tried to link the expression level of PD-1 to immune responses to checkpoint inhibitors. But some tumors with very low level of PD-1 still respond to the drugs. Though definitive data are lacking, current evidence suggests that patient with high mutational burden in tumors may have a higher chance of responding to immunotherapies. Take one step back; the long lasting, recurrentfree effect of immunotherapy implicates its advantage in controlling cancer stem cells (CSCs), which are the main cause of cancer relapse and failure of traditional cancer therapies. A deeper understanding on the properties and mechanisms underlying regulation of success regulation of CSCs by immunotherapy may shed light on delivering safer and more effective therapeutic strategies to more patients.

\section{Acknowledgements}

None.

\section{Conflict of interest}

The author declares no conflict of interest. 


\section{References}

1. World Health Organization. Cancer Key factors. Switzerland; WHO; 2017.

2. Hanahan D, Weinberg RA. Hallmarks of cancer: the next generation. Cell. 2011;144(5):646-674.
3. Brown CE, Alizadeh D, Starr R, et al. Regression of glioblastoma after chimeric antigen receptor T-Cell therapy. $N$ Engl J Med. 2016;375(26):2561-2569.

4. Kang J, Demaria S, Formenti S. Current clinical trials testing the combination of immunotherapy with radiotherapy. $J$ Immunother Cancer. $2016 ; 4: 51$ 